\title{
Production of Biogas by Using Different Pretreatments of Rice Straw Under Aerobic and Semi Aerobic Conditions.
}

Ramy Mosaad El-Khayat ${ }^{1}$; Frahat Foda Ali²; Salah M.M. Saad²; Hamed El-Sayed Abou-Aly²; Azmy N. Estefanous ${ }^{1}$.

${ }^{1}$ Soils , water and Environ.Res.Inst.,Agric.Res.Center,Giza,Egypt

${ }^{2}$ Fac. of Agric., Benha University, Moshtohor, Egypt.

Corresponding author: farhat.fouda@fagr.bu.edu.eg

\begin{abstract}
Hared lignocellulosic structure of rice straw is the main hindrance in biogas production process. Thus, laboratory scale batch experiment was conducted to study the effect of different pretreatments of rice straw on anaerobic digestion. For this reasons, different pretreatments of rice straw prior anaerobic digestion was run using chemical activator, cattle dung, Tricoderma viride, phanerochaete chrysosporium and effective microorganisms under aerobic and semi aerobic conditions to study the effect of these pretreatments on the speed and production of biogas. The results indicated that semi aerobic pretreatment has significantly increased the production of biogas more than aerobic pretreatments. The highest yield of biogas and methane production were obtained after anaerobic digestion process at three days from pretreatments. Pretreatment T4 (rice straw + cattle dung + effective microorganisms + starter) achieved in semi aerobic in biogas and methane higher than aerobic pretreatments where, obtained 9.37, 5.94 L biogas and 5.42, 3.22 L methane, respectively. Biogas and methane production rates were based on either total solid or volatile solid where, ranged between $46.60-381.83 \mathrm{~L} / \mathrm{Kg}$ consumed biogas and 18.98- $220.86 \mathrm{~L} / \mathrm{Kg}$ consumed methane. Volatile fatty acids concentration were increased during pretreatments to reach the highest concentration after three days, then decreased subsequently, meanwhile the concentration decreased after anaerobic fermentation process. Also, the $\mathrm{pH}$ values increased at the end of anaerobic digestion the values ranged between 7.02-8.28. Ammonical- $\mathrm{N}$ concentration was decreased during pretreatments to reach its lowest the concentration after seven days, then decreased at the end of fermentation process where, ranged between $23-138 \mathrm{mg} / \mathrm{Kg}$ in final anaerobic digestion process. Pretreatment T4B3 (rice straw + cattle dung + effective microorganisms + starter) after three days from semi aerobic led to the best biogas manure which contents much humic substances.
\end{abstract}

Keywords: Biogas, lignocellulosic , anaerobic digestion, microorganisms , aerobic and semi aerobic pretreatment, fermentation process

\section{Introduction}

The amount of agricultural wastes in Egypt represents in recent years, about 116.5 million tons / year whether they are plant or animal residues (ADP, 2016). Rice straw is a major agricultural waste and the amount rice straw reached approximately 3.12 million ton per year in Egypt (MALR, 2015). Lignocellulosic biomass of rice straw was contains about 19-27\% hemicellulose, 32-47\% cellulose, and 5-24\% lignin, and is difficult to digest (Liu et al., 2018). The most traditional utilization of rice by-products incorporate straw and hull for energy production of biogas through anaerobic digestion, animal feed, building materials and paper make. A stand by to reusing such waste is the compost production through microbial activity (Ali, 2011).

Anaerobic digestion is a complex biochemical process amid which organic materials are breakdown by a consortium of microbial, without oxygen, bringing about the generation of biogas (Lim et al., 2018).

Zhou et al. (2012) revealed that microbial pretreatment of corn stalks was done and it was discovered that corn straws pretreated with cow dung and sludge produced $19.6 \%$ and $18.9 \%$ higher cumulative biogas yield separately when contrasted with untreated straws by solid state anaerobic digestion performed for 60 days.
Ariunbaatar et al. (2014) found that the grinding, milling just as homogenization forms act to build the particular surface territory and uncover the cell segments of the natural waste for microbial degradation. An intricate surface area increment the substrate bacteria contact which accelerate the anaerobic digestion of organic solid waste.

Jönsson and Martin (2016) found that biological pretreatment of anaerobic digestion substrates is done under both aerobic and anaerobic conditions a longside addition of specific enzymes, for example, lipases, peptidases, cellulases among others to the anaerobic digestion process.

Awe et al. (2017) reported that biogas is worthy renewable energy with secondary energy carrier and its make can be achieved from biodegradable organic materials and it is essentially consist of gases of methane $\left(\mathrm{CH}_{4}\right)$, carbon dioxide $\left(\mathrm{CO}_{2}\right)$, less amount of hydrogen sulphide $\left(\mathrm{H}_{2} \mathrm{~S}\right)$ gas with some amount of dampness and siloxanes. Synthesis of biogas with percentage of every constituent gas is $45-70 \%$ of methane $\left(\mathrm{CH}_{4}\right), 30-40 \%$ of carbon dioxide gas $\left(\mathrm{CO}_{2}\right)$ and $1-1.5 \%$ of nitrogen $\left(\mathrm{N}_{2}\right)$ gas as major components and it is differ in compositions dependent on source of biogas production (sewage digester, organic residues digester and landfill sources). In production of chemicals or biochemical, hydrogen and synthesis gas, biogas is utilized as beginning material or fuel sources. 
Yıldırım et al. (2017) found that biological pretreatment is for the most part connected with the utilization of biological operators or fungal species that produce enzymes capable of breakdown the solid organic wastes.

\section{Materials and Methods}

Rice straw was collected from Moshtohor surrounding Farms, Kalubia Governorate, Egypt. It was air dried and hacked to little pieces $(3-5 \mathrm{~cm})$ before pretreatment to give a huge surface for liquid adhesion and direct contact with microorganisms. Fresh cattle dung was gathered from the farms of Faculty of Agriculture farm, Moshtohor, Kalubia Governorate. It was utilized as fresh as possible inside 1-2 days. Starter was taken from an old working biogas digester at Training Center for Recycling of Agricultural Residues (TCRAR), Agric, Res. Center at Moshtohor, Kalubia Governorate. Effective microorganisms (EM) were provided from Ministry of State for Environmental Affairs. Trichoderma viride strain (NRRL3635) and Phanerochaete chrysosporium strain (NRRL3659) were acquired from Microbiology Department, Soils, Water and Environment Research Inst. (SWRI). Agric. Res. Center (ARC), Giza, Egypt. While, Trichoderma viride was used as cellulose decomposers (Tom Volk, 2004) and Phanerochaete chrysosporium was utilized as lignin decomposers (Sheldon et al., 2008). All chemicals and salts were kindly provided by Sigmaaldrith Co.

\section{Preparation of inoculants:}

Flasks containing $150 \mathrm{ml}$ of sterilized CzapeckDox medium for both Trichoderma viride and Phanerochaete chrysosporium (Difco, 1977) then, inoculated and incubated at a rotary shaker (180 rpm) at $28^{\circ} \mathrm{C}$ for 5 days. Fresh preparations of cultures were centrifuging to get cells and resuspended in a similar volume of water to give a concentration of cell forming unit (cfu) $10^{8} / \mathrm{ml}$. The inocula were applied at $100 \mathrm{ml} / \mathrm{kg}$ pretreatment materials.

Experimental procedure:

This experiment was set up to assess the impact of the different pretreatments on biogas production and speed of production gas from rice straw. This experiment was carried out at Biogas Training Center, Moshtohour, SWRI, ARC. Four treatments were prepared as follows:

T1: $1650 \mathrm{~g}$ rice straw $+15 \mathrm{~g}\left(\mathrm{NH}_{4}\right)_{2} \mathrm{SO}_{4}+15 \mathrm{~g}$ $\mathrm{KH}_{2} \mathrm{PO}_{4}+6$ liter water.

$\mathrm{T} 2: 1650 \mathrm{~g}$ rice straw $+3 \mathrm{Kg}$ fresh cattle dung +6 liter water.

T3: T2 $+100 \mathrm{ml} \mathrm{T.} \mathrm{viride} \mathrm{and} \mathrm{P.} \mathrm{chrysosporium.}$

T4: T2 + 100 ml Effective microorganisms (EM).

The previous treatments were fermentation in plastic containers with holes on each side separately for aerobic pretreatments and one side for semi aerobic pretreatments. Samples were taken after 0, 1,
3, 5 and 7 days from all these treatments (100 g) and mixed with $700 \mathrm{ml}$ starter to produce gas production.

The evolved biogas was daily measured, while its contents of $\mathrm{CH}_{4}$ were estimated every day throughout the experimental periods. The physical and chemical properties of rice straw were determined. Also, microbiological determinations such as cellulose decomposers and acid producing bacteria (aerobic and anaerobic) were estimated at initial and at the end of the fermentation periods.

Analytical methods:

Daily biogas yield was estimated according to (Maramba et al., 1978). Methane content was determined by gas- liquid chromatography according to Wujick and Jewell (1980). Total solids (TS), volatile solids (VS), organic carbon (O.C), total phosphorus (TP), total potassium (TK) and total volatile fatty acids (VFAs) were determined according to the standard method recommended by APHA (1992). Moisture content (M.C), organic matter (O.M), ammoniacal nitrogen $\mathrm{NH}_{4}-\mathrm{N}$ and nitrate nitrogen $\left(\mathrm{NO}_{3}-\mathrm{N}\right)$ were determined according to the standard method recommended by Page et al. (1982). The $\mathrm{pH}$ values were determined according to Jodice et al. (1982). Electrical conductivity (EC) was estimated according to Richards (1954). Cellulose, lignin and hemicellulose percentages were determined according to the method described by (Rama, 2013). The percentage of loss in the TS as well as biogas and methane as $(\mathrm{L} / \mathrm{Kg})$ TS (added or consumed) and VS (added or consumed) were calculated according to the following equation to Abdel- Fatah (2012):

$\%$ TS Losses $=\left(\left(\mathrm{TS}(\mathrm{g})_{\text {Initial }}-\mathrm{TS}(\mathrm{g})_{\text {Final }}\right) / \mathrm{TS}(\mathrm{g})\right.$ Initial) $\times 100$

$\mathrm{TS}$ or $\mathrm{VS}_{\text {added }}(\mathrm{L} / \mathrm{Kg})$ biogas $=$ Total liter biogas $/$ $\mathrm{TS}$ or VS $(\mathrm{Kg})$ Initial

TS added or VS $(\mathrm{L} / \mathrm{Kg})$ methane = Total liter methane / TS or VS (Kg) Initial

$\mathrm{TS}$ or $\mathrm{VS}$ consumed $(\mathrm{L} / \mathrm{Kg})$ biogas $=$ Total liter biogas / TS or VS Losses (Kg)

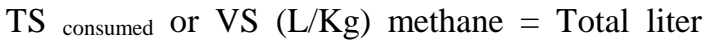
methane / TS or VS Losses (Kg)

Acid producing bacteria were counted on nutrient broth medium according to Cunningham (1954) using Most Probable Number (MPN) technique. Aerobic and anaerobic cellulose decomposing bacteria were determined according to Cochran (1950) using most probable number technique (MPN). Statistical analysis:

All data collected were subjected to analysis of variance to test treatment effects for significance using Analytical software (2013). The means were compared using F-LSD according to Snedecor and Cochran (1991). The models used for statistical analyses were three ways factorial of variance was used to test the effect of different treatments, pretreatments (aerobic and semi aerobic), period of day and the interaction between both. 
Results and Discussion

Chemical and physical characteristics of rice straw, cattle dung and starter

The chemical and physical analysis of the used rice straw, cattle dung and starter for biogas production were illustrated in Table (1). The obtained resulted showed that the percentages of moisture content, total solids, organic matter, organic carbon, total nitrogen, total phosphorus and total nitrogen were found to be $(7.84,78.04,90.00 \%),(92.16,21.96,10.00 \%),(75.89$, $58.41,50.87 \%),(44.02,33.88,29.50 \%),(0.64,1.13$, $1.64 \%),(0.06,0.60,0.52 \%)$ and $(1.03,0.46,0.73 \%)$, respectively for rice straw, cattle dung and starter. On the other hand, the percentage of cellulose, lignin and hemicellulose were $40.54,8.92$ and $27.11 \%$ for rice straw and $24.17,18.16$ and $16.08 \%$ for cattle dung, respectively. But, the ash contents were found to be $24.11,41.59$ and $49.13 \%$ for rice straw, cattle dung and starter, respectively. Also, these results showed that the percentage of ammonical in starter (521 $\mathrm{mg} / \mathrm{kg}$ ) was more than cattle dung $(444.46 \mathrm{mg} / \mathrm{kg})$ and lower than rice straw $(35.90 \mathrm{mg} / \mathrm{kg})$. However, the values of volatile fatty acids in rice straw, cattle dug and starter were $1.84,11.36$ and $5.00 \mathrm{meq} / \mathrm{Kg}$, respectively. The acquired results are in agreement with those reported by Liu et al, (2018), Estefanous et al. (2010) and El-Akshar et al. (2012).

Table 1. Chemical and physical characteristics of rice straw, cattle dung and starter.

\begin{tabular}{lccc}
\hline \multicolumn{1}{c}{ Components } & Rice straw & Cattle dung & Starter \\
\hline Moisture content \% & 7.84 & 78.04 & 90.00 \\
Total solids \% & 92.16 & 21.96 & 10.00 \\
$\mathrm{pH}(1: 10)$ & 6.80 & 7.32 & 7.22 \\
EC (1:10) dS/m & 4.59 & 4.18 & 4.03 \\
Ammoniacal -N (mg/Kg) & 35.90 & 444.46 & 521 \\
Nitrate -N (mg/Kg) & 7.00 & 24.70 & 5.00 \\
Organic matter (O.M) \% & 75.89 & 58.41 & 50.87 \\
Ash \% & 24.11 & 41.59 & 49.13 \\
Organic carbon \% & 44.02 & 33.88 & 29.50 \\
Total nitrogen \% & 0.64 & 1.13 & 1.64 \\
C/N ratio & $68.78: 1$ & $29.98: 1$ & $17.98: 1$ \\
Total phosphorus (\%) & 0.06 & 0.60 & 0.52 \\
Total potassium $(\%)$ & 1.03 & 0.46 & 0.73 \\
Volatile fatty acid (meq/kg) & 1.84 & 11.36 & 5.00 \\
Cellulose \% & 40.54 & 24.17 & - \\
Lignin \% & 8.92 & 18.16 & - \\
Hemicellulose \% & 27.11 & 16.08 & - \\
\hline
\end{tabular}

Changes of total solids during pretreatments of rice straw and anaerobic digestion at 50 days

The changes of total solids (TS) during both pretreatments of rice straw and anaerobic digestion at 50 days are shown in Table (2). The results showed that during anaerobic digestion the amount of TS were reduced in all treatments. It was ranged between 97.85 $-140.43 \mathrm{~g}$ after anaerobic digestion. The loss percentage of TS after anaerobic digestion ranged between $6.38-34.95 \%$ of TS. These decrements may be due to the total solids occurred in the form of gases and water. The accomplished results are in agreement with those reported by EL- Akshar (2000), found that the concentration of TS decreased through anaerobic digestion and such loss in TS occurred in the form gases and water and the losses in case of Jews mallow processing higher than cattle dung. The decrement is a normal response to microbial oxidation of a part of the organic carbon. Also, Estefanous et al. (2010) reported that during anaerobic digestion TS was reduced may be due to a high substrate conversion rated to $\mathrm{CO}_{2}$ and $\mathrm{CH}_{4}$ in the anaerobic digestion. Ugwuoke et al. (2015) mentioned that total solid of any given biogas feedstock specifically contributes to the execution of the system and accummulative of biogas amid anaerobic digestion. There is a reverse connection between the total solid content and accumulative of biogas

Changes of volatile fatty acids as (total \&acetic acid), amonical nitrogen and $\mathrm{pH}$ values during pretreatments of rice straw and anaerobic digestion at 50 days

Data in Table (3) show the change of volatile fatty acid as (total \&acetic acid), $\mathrm{NH}_{4}-\mathrm{N}$ and $\mathrm{pH}$ values during the different pretreatment processes of rice straw and anaerobic digestion at 50 days. Volatile fatty acids either total or acetic acid obtained the best value after three days from semi aerobic pretreatment which recorded values greater than aerobic pretreatment $(60.40 \mathrm{meq} / \mathrm{L}$ and $5177.14 \mathrm{mg} / \mathrm{Kg})$, respectively with $\mathrm{T} 4$. On the other hand, T1 achieved the worst values where recorded $19.20 \mathrm{meq} / \mathrm{L}$ and $1645.71 \mathrm{mg} / \mathrm{Kg}$ after seven days, respectively. Then VFAs decreased after anaerobic digestion this decrement may be due to its consumption by methane bacteria during anaerobic digestion. The obtained results were similar to those results obtained by ElAkshar (2000), Estefanous (1993) and Zhange et al. (2014) who reported that the concentration of VFAs decreased by increasing the fermentation may be due 
to the VFAs which were converted into $\mathrm{CO}_{2}$ and $\mathrm{CH}_{4}$ by methane bacteria.

$\mathrm{pH}$ values were low after pretreatments; it ranged between $6.44-7.73$ in all treatments, this may be due to the high activity of the acid producing bacteria. Then, $\mathrm{pH}$ values increased after anaerobic digestion, it ranged between $7.02-8.28$ in all treatments. This may be due to $\mathrm{CaCO}_{3}$ buffer in the initial wastes mixture and the natural decomposition of this material. These results were similar to those reported by Budiyono et al. (2010), Estefanous et al. (2010) and Maria et al. (2019).

The result in Table (3) presented that $\mathrm{NH}_{4}-\mathrm{N}$ concentration was high at the initial pretreatment processes. It ranged between $188-369 \mathrm{mg} / \mathrm{Kg}$ in all treatments. Then decreased in all treatments to reach the lowest concentration after 7 days from aerobic or semi aerobic pretreatment. It ranged between 108-326 $\mathrm{mg} / \mathrm{Kg}$. The concentration of $\mathrm{NH}_{4}-\mathrm{N}$ was decreased after anaerobic digestion. It ranged between 23- 138 $\mathrm{mg} / \mathrm{Kg}$. These decrements may be due to either volatilization or trans formation to organic nitrogen as bacteria cells. These results are in agreement with those reported by. Estefanous et al. (2010) found that the concentration of $\mathrm{NH}_{4}-\mathrm{N}$ increased along with increased anaerobic digestion to reach it's the highest values after four weeks, being $1200 \mathrm{mg} / \mathrm{Kg}$ and then decreased to reach $325 \mathrm{mg} / \mathrm{Kg}$ after 8 weeks of digestion.

Table 2. Changes of total solids during pretreatments of rice straw and anaerobic digestion at 50 days

\begin{tabular}{|c|c|c|c|c|c|c|}
\hline \multirow{3}{*}{ Treatments } & \multirow{3}{*}{ Conditions } & \multirow{3}{*}{$\begin{array}{c}\text { Periods } \\
\text { (Days) }\end{array}$} & \multicolumn{4}{|c|}{ Total solids (TS) } \\
\hline & & & & & & \\
\hline & & & Initial & Final & $\mathrm{g}$ & $\%$ \\
\hline \multirow{9}{*}{ 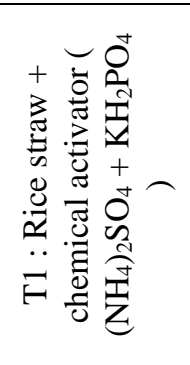 } & \multirow{5}{*}{$\underset{\frac{0}{2}}{\frac{0}{0}}$} & 0 & 150.00 & 97.85 & 52.15 & 34.77 \\
\hline & & 1 & 150.00 & 97.58 & 52.42 & 34.95 \\
\hline & & 3 & 150.00 & 119.84 & 30.16 & 20.11 \\
\hline & & 5 & 150.00 & 122.75 & 27.25 & 18.17 \\
\hline & & 7 & 150.00 & 126.57 & 23.43 & 15.62 \\
\hline & \multirow{4}{*}{ 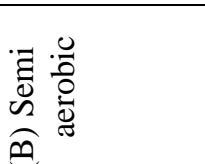 } & 1 & 150.00 & 113.27 & 36.73 & 24.49 \\
\hline & & 3 & 150.00 & 112.43 & 37.57 & 25.05 \\
\hline & & 5 & 150.00 & 116.86 & 33.14 & 22.09 \\
\hline & & 7 & 150.00 & 124.57 & 25.43 & 16.95 \\
\hline \multirow{9}{*}{ 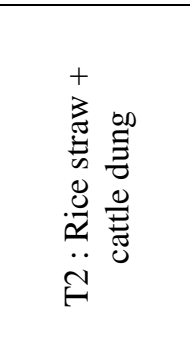 } & \multirow{5}{*}{$\underset{\frac{\pi}{2}}{\frac{0}{0}}$} & 0 & 150.00 & 126.98 & 23.02 & 15.35 \\
\hline & & 1 & 150.00 & 126.11 & 23.89 & 15.93 \\
\hline & & 3 & 150.00 & 129.09 & 20.91 & 13.94 \\
\hline & & 5 & 150.00 & 128.95 & 21.05 & 14.03 \\
\hline & & 7 & 150.00 & 131.76 & 18.24 & 12.16 \\
\hline & \multirow{4}{*}{ 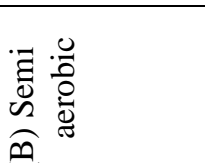 } & 1 & 150.00 & 105.29 & 44.71 & 29.81 \\
\hline & & 3 & 150.00 & 114.94 & 35.06 & 23.37 \\
\hline & & 5 & 150.00 & 119.74 & 30.26 & 20.17 \\
\hline & & 7 & 150.00 & 124.45 & 25.55 & 17.03 \\
\hline \multirow{9}{*}{ 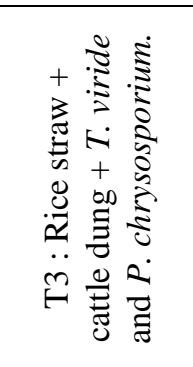 } & \multirow{5}{*}{ 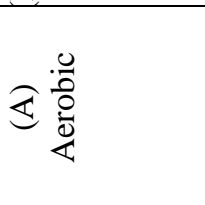 } & 0 & 150.00 & 119.34 & 30.66 & 20.44 \\
\hline & & 1 & 150.00 & 118.56 & 31.44 & 20.96 \\
\hline & & 3 & 150.00 & 121.44 & 28.56 & 19.04 \\
\hline & & 5 & 150.00 & 127.67 & 22.33 & 14.89 \\
\hline & & 7 & 150.00 & 126.84 & 23.16 & 15.44 \\
\hline & \multirow{4}{*}{ 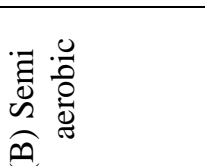 } & 1 & 150.00 & 111.32 & 38.68 & 25.79 \\
\hline & & 3 & 150.00 & 118.39 & 31.61 & 21.07 \\
\hline & & 5 & 150.00 & 122.96 & 27.04 & 18.03 \\
\hline & & 7 & 150.00 & 127.52 & 22.48 & 14.99 \\
\hline \multirow{9}{*}{ 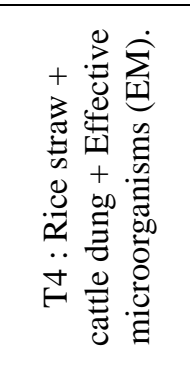 } & \multirow{5}{*}{ 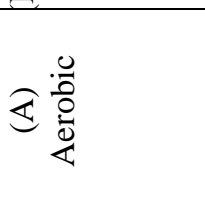 } & 0 & 150.00 & 105.04 & 44.96 & 29.97 \\
\hline & & 1 & 150.00 & 118.03 & 31.97 & 21.31 \\
\hline & & 3 & 150.00 & 123.03 & 26.97 & 17.98 \\
\hline & & 5 & 150.00 & 125.16 & 24.84 & 16.56 \\
\hline & & 7 & 150.00 & 140.43 & 9.57 & 6.38 \\
\hline & \multirow{4}{*}{ 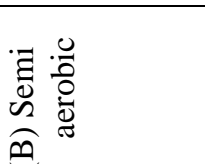 } & 1 & 150.00 & 126.36 & 23.64 & 15.76 \\
\hline & & 3 & 150.00 & 125.46 & 24.54 & 16.36 \\
\hline & & 5 & 150.00 & 125.86 & 24.14 & 16.09 \\
\hline & & 7 & 150.00 & 127.67 & 22.33 & 14.89 \\
\hline \multirow{3}{*}{ L.S.D (0.05) } & \multicolumn{2}{|c|}{ Treatment } & & 0.2316 & 0.2683 & 0.2496 \\
\hline & \multirow{2}{*}{\multicolumn{2}{|c|}{ Pretreatment }} & & 0.1638 & 0.1897 & 0.1765 \\
\hline & & & & 0.7323 & 0.8483 & 0.7893 \\
\hline
\end{tabular}


Table 3. Changes of volatile fatty acids (as total \&acetic acid), ammonical nitrogen and $\mathrm{pH}$ during pretreatments of rice straw and anaerobic digestion at 50 days

\begin{tabular}{|c|c|c|c|c|c|c|c|c|c|c|}
\hline \multirow{3}{*}{ 苞 } & \multirow{3}{*}{ 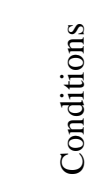 } & \multirow{3}{*}{$\begin{array}{c}\text { Periods } \\
\text { (Days) } \\
\text { (Days) }\end{array}$} & \multicolumn{4}{|c|}{ Volatile fatty acid } & \multirow{2}{*}{\multicolumn{2}{|c|}{$\mathrm{pH}$}} & \multirow{2}{*}{\multicolumn{2}{|c|}{$\begin{array}{c}\mathrm{NH}_{4}-\mathrm{N} \\
(\mathrm{mg} / \mathrm{Kg})\end{array}$}} \\
\hline & & & \multicolumn{2}{|c|}{$\begin{array}{c}\text { Total } \\
(\mathrm{meq} / \mathrm{L})\end{array}$} & \multicolumn{2}{|c|}{$\begin{array}{c}\text { Acetic acid } \\
(\mathrm{mg} / \mathrm{Kg})\end{array}$} & & & & \\
\hline & & & Initial & Final & Initial & Final & $\begin{array}{c}\text { Initia } \\
1\end{array}$ & Final & Initial & Final \\
\hline \multirow{18}{*}{ 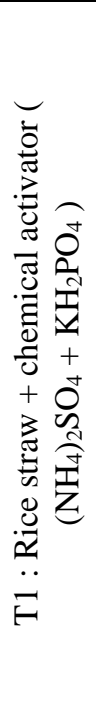 } & \multirow{10}{*}{ 至 $\frac{0}{0}$} & 0 & 20.80 & 15.20 & 1782.86 & 1302.86 & 6.44 & 7.02 & 222.0 & 95.00 \\
\hline & & 1 & 22.40 & 14.40 & 1920.00 & 1234.29 & 7.18 & 7.39 & 0 & 67.00 \\
\hline & & 3 & 24.80 & 10.80 & 2125.71 & 925.71 & 7.10 & 7.55 & 180.0 & 60.00 \\
\hline & & 5 & 23.60 & 11.60 & 2022.86 & 994.29 & 7.00 & 7.41 & 0 & 63.00 \\
\hline & & 7 & 19.20 & 17.20 & 1645.71 & 1474.29 & 7.28 & 7.83 & 176.0 & 97.00 \\
\hline & & & & & & & & & 0 & \\
\hline & & & & & & & & & 148.0 & \\
\hline & & & & & & & & & 0 & \\
\hline & & & & & & & & & 121.0 & \\
\hline & & & & & & & & & $\frac{0}{2(0)}$ & \\
\hline & \multirow{8}{*}{ 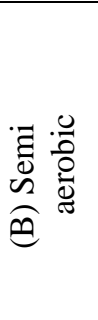 } & 1 & 30.00 & 16.00 & 2571.43 & 1371.43 & 7.50 & 8.01 & 369.0 & 59.00 \\
\hline & & 3 & 36.00 & 13.20 & 3085.71 & 1131.43 & 7.50 & 8.19 & 0 & 47.00 \\
\hline & & 5 & 33.20 & 14.80 & 2845.71 & 1268.57 & 7.43 & 8.05 & 345.0 & 53.00 \\
\hline & & 7 & 26.80 & 16.80 & 2297.14 & 1440.00 & 7.72 & 8.00 & 0 & 67.00 \\
\hline & & & & & & & & & 336.0 & \\
\hline & & & & & & & & & 0 & \\
\hline & & & & & & & & & 326.0 & \\
\hline & & & & & & & & & 0 & \\
\hline \multirow{13}{*}{ 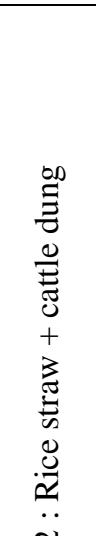 } & \multirow{10}{*}{ 离 $\frac{.0}{0}$} & 0 & 22.40 & 14.80 & 1920.00 & 1268.57 & 6.70 & 7.19 & 188.0 & 107.00 \\
\hline & & 1 & 24.00 & 14.00 & 2057.14 & 1200.00 & 7.46 & 7.75 & 0 & 74.00 \\
\hline & & 3 & 26.40 & 9.20 & 2262.86 & 788.57 & 7.20 & 7.63 & 185.0 & 50.00 \\
\hline & & 5 & 25.60 & 10.80 & 2194.29 & 925.71 & 7.17 & 7.53 & 0 & 57.00 \\
\hline & & 7 & 20.40 & 16.40 & 1748.57 & 1405.71 & 7.24 & 7.87 & 153.0 & 83.00 \\
\hline & & & & & & & & & 0 & \\
\hline & & & & & & & & & 153.0 & \\
\hline & & & & & & & & & 0 & \\
\hline & & & & & & & & & 109.0 & \\
\hline & & & & & & & & & 0 & \\
\hline & \multirow{8}{*}{ 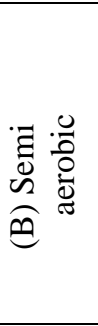 } & 1 & 39.60 & 15.60 & 3394.29 & 1337.14 & 7.44 & 8.11 & 352.0 & 49.00 \\
\hline & & 3 & 45.20 & 12.00 & 3874.29 & 1028.57 & 7.46 & 8.23 & 0 & 33.00 \\
\hline & & 5 & 40.80 & 12.40 & 3497.14 & 1062.86 & 7.55 & 8.15 & 359.0 & 44.00 \\
\hline $\mathcal{N}$ & & 7 & 33.60 & 16.40 & 2880.00 & 1405.71 & 7.53 & 8.04 & 0 & 53.00 \\
\hline & & & & & & & & & 314.0 & \\
\hline & & & & & & & & & 0 & \\
\hline & & & & & & & & & 321.0 & \\
\hline & & & & & & & & & 0 & \\
\hline \multirow{16}{*}{ 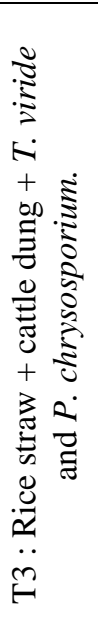 } & \multirow{10}{*}{ ¿ $\frac{0}{0}$} & 0 & 23.20 & 14.00 & 1988.57 & 1200.00 & 6.61 & 7.18 & 203.0 & 116.00 \\
\hline & & 1 & 25.60 & 13.60 & 2194.29 & 1165.71 & 7.50 & 7.81 & 0 & 55.00 \\
\hline & & 3 & 27.60 & 8.40 & 2365.71 & 720.00 & 7.19 & 7.57 & 183.0 & 45.00 \\
\hline & & 5 & 26.80 & 10.40 & 2297.14 & 891.43 & 7.36 & 7.71 & 0 & 51.00 \\
\hline & & 7 & 22.00 & 15.60 & 1885.71 & 1337.14 & 7.16 & 7.77 & 148.0 & 67.00 \\
\hline & & & & & & & & & 0 & \\
\hline & & & & & & & & & 150.0 & \\
\hline & & & & & & & & & 0 & \\
\hline & & & & & & & & & 108.0 & \\
\hline & & & & & & & & & 0 & \\
\hline & & 1 & 42.00 & 15.20 & 3600.00 & 1302.86 & 7.48 & 8.09 & 338.0 & 41.00 \\
\hline & & 3 & 54.80 & 10.80 & 4697.14 & 925.71 & 7.53 & 8.25 & 0 & 29.00 \\
\hline & $\exists \cdot \tilde{0}$ & 5 & 49.60 & 11.60 & 4251.43 & 994.29 & 7.50 & 8.17 & 347.0 & 37.00 \\
\hline & $\ddot{\infty}$ & 7 & 38.40 & 16.00 & 3291.43 & 1371.43 & 7.44 & 8.06 & 0 & 49.00 \\
\hline & $\widehat{\ominus}^{\pi}$ & & & & & & & & 314.0 & \\
\hline & & & & & & & & & 0 & \\
\hline
\end{tabular}




\begin{tabular}{|c|c|c|c|c|c|c|c|c|c|c|}
\hline & & & & & & & & & $\begin{array}{c}317.0 \\
0\end{array}$ & \\
\hline \multirow{17}{*}{ 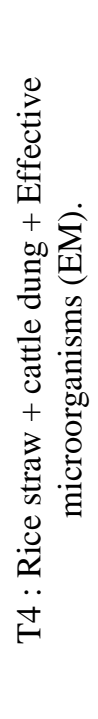 } & \multirow{9}{*}{$\underset{\&}{\frac{0}{2}} \frac{0}{0}$} & 0 & 24.40 & 12.40 & 2091.43 & 1062.86 & 6.60 & 7.16 & 208.0 & 138.00 \\
\hline & & 1 & 27.20 & 12.00 & 2331.43 & 1028.57 & 7.44 & 7.73 & 0 & 121.00 \\
\hline & & 3 & 30.00 & 7.60 & 2571.43 & 651.43 & 7.23 & 7.73 & 207.0 & 30.00 \\
\hline & & 5 & 28.40 & 9.60 & 2434.29 & 822.86 & 7.57 & 7.90 & 0 & 37.00 \\
\hline & & 7 & 24.00 & 14.80 & 2057.14 & 1268.57 & 7.10 & 7.86 & 146.0 & 64.00 \\
\hline & & & & & & & & & 0 & \\
\hline & & & & & & & & & 158.0 & \\
\hline & & & & & & & & & 0 & \\
\hline & & & & & & & & & 113.0 & \\
\hline & \multirow{8}{*}{ 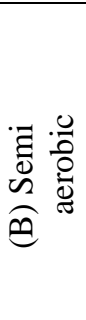 } & 1 & 46.80 & 12.40 & 4011.43 & 1062.86 & 7.50 & 8.14 & 328.0 & 37.00 \\
\hline & & 3 & 60.40 & 8.40 & 5177.14 & 720.00 & 7.59 & 8.28 & 0 & 23.00 \\
\hline & & 5 & 52.40 & 10.40 & 4491.43 & 891.43 & 7.73 & 8.21 & 331.0 & 31.00 \\
\hline & & 7 & 43.20 & 15.20 & 3702.86 & 1302.86 & 7.54 & 8.11 & 0 & 40.00 \\
\hline & & & & & & & & & 331.0 & \\
\hline & & & & & & & & & 0 & \\
\hline & & & & & & & & & 290.0 & \\
\hline & & & & & & & & & 0 & \\
\hline \multirow{6}{*}{$\begin{array}{l}\text { L.S.D } \\
(0.05)\end{array}$} & \multirow{2}{*}{\multicolumn{2}{|c|}{ Treatment }} & 0.525 & 0.4709 & & & & & & \\
\hline & & & 0 & & & & & & & \\
\hline & \multirow{2}{*}{\multicolumn{2}{|c|}{ Pretreatment }} & 0.371 & 0.3330 & & & & & & \\
\hline & & & 2 & & & & & & & \\
\hline & \multirow{2}{*}{\multicolumn{2}{|c|}{ Period of day }} & 1.660 & 1.4891 & & & & & & \\
\hline & & & 1 & & & & & & & \\
\hline
\end{tabular}

$\overline{\text { Changes of cellulose decomposers bacteria (aerobic and anaerobic) during pretreatments of rice straw and anaerobic digestion }}$ at 50 days

The numbers of aerobic and anaerobic cellulose decomposing bacteria during different pretreatment processes of rice straw with additional supplements and anaerobic digestion at 50 days are presented in Table(4). Data showed that the numbers of aerobic and anaerobic cellulose decomposing bacteria were increased in all treatments to reach its highest values after 7 days from aerobic and semi anaerobic pretreatments. The numbers of aerobic cellulose decomposing bacteria was decreased after anaerobic digestion in all treatments. On the other hand, the numbers of anaerobic cellulose decomposing bacteria were increased after anaerobic digestion in all treatments. The obtained results were recorded by ElAkshar (2000) reported that the early proliferation of anaerobic cellulose decomposers was accompanied by the depletion of oxygen and presence of ample supply of cellulosic materials.

Changes of acid producing bacteria during pretreatments of rice straw and anaerobic digestion at 50 days

Behavior of aerobic and anaerobic acid producing bacteria during the different pretreatment processes of rice straw with additional supplements and anaerobic digestion at 50 days are presented in Table (4). The initial counts of aerobic and anaerobic acid producing bacteria ranged between $0.01 \times 10^{6}$ and $506 \times 10^{6}$ MPN/g.D.W for aerobic producing bacteria and $0.03 \times 10^{6}$ and $1003 \times 10^{6}$ for anaerobic acid producing bacteria from zero time to one day. The numbers of aerobic and anaerobic acid producing bacteria were increased in all treatments to reach the highest values after 7 days from aerobic and semi anaerobic pretreatments. The numbers of aerobic and anaerobic acid producing bacteria were decreased with all treatments after anaerobic digestion. The acquired results are in agreements with those reported by Estefanous (1993) who found the accumulation of acids and explained the decrease of their concentrations by the decrease of acids producer's numbers. This was expected as a logic consequence of the non -consuming of the products and accumulation of it which usually leads to the inhibition of their growth. Also, El-Akshar (2000) reported that anaerobic acid producing bacteria were gradually increased with the increasing of fermentation and reached their maximum records at the $21^{\text {st }}$ day and gradually decreased there after till the end of the anaerobic digestion. 
Table 4. Changes of cellulose decomposers and acid producing bacteria (aerobic and anaerobic) (MPN/g.D.W) during different pretreatment processes of rice straw and anaerobic digestion at 50 days

\begin{tabular}{|c|c|c|c|c|c|c|c|c|c|c|}
\hline \multirow{3}{*}{ 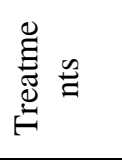 } & \multirow{3}{*}{ ن̃ } & \multirow{3}{*}{$\begin{array}{c}\text { Periods } \\
\text { (Days) }\end{array}$} & \multicolumn{4}{|c|}{ Cellulose decomposers } & \multicolumn{4}{|c|}{ Acid producers } \\
\hline & & & \multicolumn{2}{|c|}{ Aerobic $\times 10^{3}$} & \multicolumn{2}{|c|}{$\begin{array}{c}\text { Anaerobic } \\
\times 10^{3}\end{array}$} & \multicolumn{2}{|c|}{ Aerobic $\times 10^{6}$} & \multicolumn{2}{|c|}{ Anaerobic $\times 10^{6}$} \\
\hline & & & Initial & Final & Initial & Final & Initial & Final & Initial & Final \\
\hline \multirow{9}{*}{ 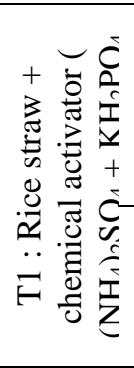 } & \multirow{5}{*}{ 㐫 } & 0 & 1.61 & 0.81 & 0.61 & 1.81 & 0.01 & 0.004 & 0.03 & 0.006 \\
\hline & & 1 & 2.13 & 1.03 & 0.66 & 1.92 & 0.41 & 0.012 & 0.18 & 0.010 \\
\hline & & 3 & 3.17 & 1.23 & 0.71 & 2.01 & 0.93 & 0.12 & 0.35 & 0.110 \\
\hline & & 5 & 3.91 & 1.50 & 1.11 & 2.13 & 1.24 & 0.81 & 0.67 & 0.140 \\
\hline & & 7 & 4.31 & 1.86 & 1.25 & 2.41 & 2.14 & 1.01 & 1.11 & 0.210 \\
\hline & \multirow{4}{*}{ 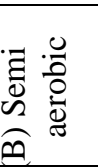 } & 1 & 1.90 & 0.03 & 12.06 & 13.17 & 1.66 & 0.31 & 117.00 & 13.00 \\
\hline & & 3 & 2.11 & 0.11 & 12.71 & 13.91 & 1.96 & 0.35 & 127.00 & 21.00 \\
\hline & & 5 & 2.66 & 0.13 & 13.91 & 14.03 & 2.36 & 0.41 & 143.00 & 43.00 \\
\hline & & 7 & 3.11 & 0.19 & 14.32 & 15.06 & 2.56 & 0.61 & 171.00 & 70.00 \\
\hline \multirow{9}{*}{ 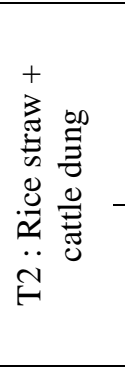 } & \multirow{5}{*}{ 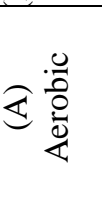 } & 0 & 13.05 & 4.18 & 2.70 & 3.18 & 1.51 & 0.17 & 1.93 & 0.18 \\
\hline & & 1 & 16.14 & 4.88 & 3.20 & 3.31 & 36.00 & 8.00 & 21.17 & 1.17 \\
\hline & & 3 & 20.11 & 5.17 & 4.08 & 5.21 & 97.00 & 11.00 & 29.11 & 1.51 \\
\hline & & 5 & 25.63 & 6.30 & 5.21 & 6.60 & 104.00 & 19.00 & 31.18 & 2.33 \\
\hline & & 7 & 29.46 & 7.01 & 6.41 & 7.91 & 121.00 & 20.00 & 35.66 & 2.45 \\
\hline & \multirow{4}{*}{ 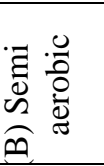 } & 1 & 2.13 & 0.66 & 25.21 & 27.01 & 390.00 & 45.00 & 700.00 & 100.00 \\
\hline & & 3 & 2.37 & 0.67 & 30.16 & 33.14 & 410.00 & 49.00 & 740.00 & 107.00 \\
\hline & & 5 & 2.80 & 0.71 & 36.73 & 40.16 & 425.00 & 61.00 & 799.00 & 130.00 \\
\hline & & 7 & 2.93 & 0.73 & 40.41 & 41.18 & 440.00 & 63.00 & 830.00 & 155.00 \\
\hline \multirow{9}{*}{ 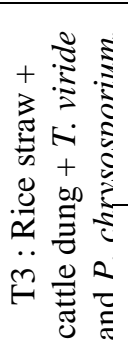 } & \multirow{5}{*}{ ¿ $\frac{0}{\frac{0}{0}} \frac{0}{0}$} & 0 & 15.18 & 4.99 & 2.99 & 4.55 & 1.60 & 0.23 & 2.81 & 0.61 \\
\hline & & 1 & 19.41 & 5.21 & 3.31 & 4.67 & 47.00 & 9.00 & 24.11 & 1.93 \\
\hline & & 3 & 23.18 & 6.19 & 4.21 & 5.90 & 103.00 & 11.70 & 33.19 & 2.11 \\
\hline & & 5 & 26.31 & 6.87 & 5.26 & 6.95 & 107.00 & 19.90 & 37.31 & 3.13 \\
\hline & & 7 & 30.04 & 7.23 & 6.71 & 8.01 & 131.00 & 24.00 & 38.39 & 3.21 \\
\hline & \multirow{4}{*}{ 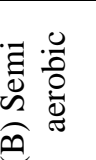 } & 1 & 3.11 & 0.91 & 33.01 & 38.07 & 470.00 & 70.00 & 900.00 & 190.00 \\
\hline & & 3 & 3.37 & 0.95 & 37.08 & 40.11 & 501.00 & 81.00 & 930.00 & 197.00 \\
\hline & & 5 & 3.41 & 1.03 & 40.11 & 42.16 & 570.00 & 82.00 & 1000.00 & 218.00 \\
\hline & & 7 & 3.60 & 1.10 & 14.16 & 47.21 & 596.00 & 91.00 & 1050.00 & 250.00 \\
\hline \multirow{9}{*}{ 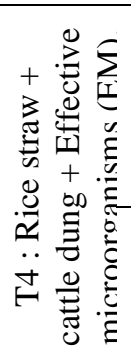 } & \multirow{5}{*}{ ¿ $\frac{0}{\frac{0}{0}} \frac{0}{0}$} & 0 & 16.14 & 5.15 & 3.01 & 5.21 & 2.50 & 0.91 & 3.31 & 1.01 \\
\hline & & 1 & 20.18 & 5.92 & 3.45 & 5.53 & 59.00 & 13.00 & 27.18 & 2.18 \\
\hline & & 3 & 25.03 & 7.11 & 4.33 & 6.33 & 114.00 & 17.00 & 36.44 & 2.61 \\
\hline & & 5 & 27.11 & 7.41 & 5.50 & 7.18 & 123.00 & 21.00 & 40.61 & 3.31 \\
\hline & & 7 & 30.51 & 7.93 & 7.04 & 8.91 & 157.00 & 25.70 & 43.21 & 4.18 \\
\hline & \multirow{4}{*}{ 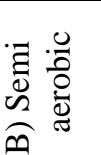 } & 1 & 6.61 & 1.23 & 43.07 & 45.61 & 506.00 & 82.00 & 1003.00 & 290.00 \\
\hline & & 3 & 7.19 & 1.27 & 47.17 & 49.31 & 610.00 & 97.00 & 1020.00 & 345.00 \\
\hline & & 5 & 7.86 & 1.40 & 47.61 & 50.06 & 645.00 & 110.00 & 1070.00 & 400.00 \\
\hline & & 7 & 8.14 & 1.43 & 49.01 & 52.66 & 670.00 & 123.00 & 1093.00 & 407.00 \\
\hline
\end{tabular}

\section{Biogas and methane production rates}

Data in Table (5\&6) show the production rates of biogas and methane during different pretreatment processes of rice straw and anaerobic digestion at 50 days. The result in Table (5) presented that biogas production obtained the highest yields of biogas after anaerobic digestion of three days from pretreatment process; semi aerobic pretreatment recorded that yields of biogas greater than aerobic pretreatment 9.37 \& 5.94 L, respectively with T4. On the other hand, T1 recorded the least yields of biogas after anaerobic digestion.

Methane production during the different pretreatment processes of rice straw and anaerobic digestion at 50 days are recorded in Table (6). It was clear that the methane production yield at the maximum total methane under anaerobic digestion after three days from pretreatment processes. Semi aerobic pretreatment achieved the total methane greater than aerobic pretreatment $5.42-3.22 \mathrm{~L}$, respectively with $\mathrm{T} 4$. On the other hand, $\mathrm{T} 1$ achieved the least total methane after anaerobic digestion. The accomplished results were similar to those obtained Abdel- Fatah (2012). She found that biogas and methane production were increased strongly with treated rice straw with cattle dung, followed by untreated rice straw mixed with cattle dung. From the obtained results, its has been shown that biogas and methane production rates greatly different according to the type of the different treatments. The biogas and methane production rates based on either TS or VS added or consumed gave the best value with treatment T4. T4 under semi aerobic condition achieved the best values treatment after three days from pretreatments 
and after anaerobic digestion. This observation may be due to attributed to the high activity of lytic microorganism. On the other hand, T1 was the least values in comparison with other treatments. The obtained results are in agreement with those reported by Hanafy et al. (2000) and Estefanous et al. (2010).

Table 5. Biogas production during different pretreatment processes of rice straw and anaerobic digestion at 50 days

\begin{tabular}{|c|c|c|c|c|c|c|c|}
\hline \multirow[b]{2}{*}{ Treatments } & \multirow[b]{2}{*}{ Conditions } & \multirow[b]{2}{*}{$\begin{array}{c}\text { Periods } \\
\text { (Days) }\end{array}$} & \multicolumn{5}{|c|}{ Biogas production } \\
\hline & & & $\begin{array}{c}\mathrm{TS} \\
\text { added } \\
(\mathrm{L} / \mathrm{Kg})\end{array}$ & $\begin{array}{c}\text { TS } \\
\text { consumed } \\
(\mathrm{L} / \mathrm{Kg}) \\
\end{array}$ & $\begin{array}{c}\text { VS } \\
\text { added } \\
(\mathrm{L} / \mathrm{Kg})\end{array}$ & $\begin{array}{c}\text { VS } \\
\text { consumed } \\
(\mathrm{L} / \mathrm{Kg}) \\
\end{array}$ & $\begin{array}{l}\text { Total } \\
\text { Liter }\end{array}$ \\
\hline \multirow{9}{*}{ 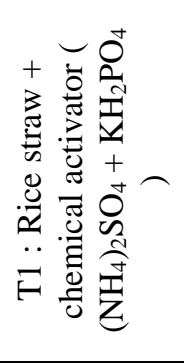 } & \multirow{5}{*}{$\underset{\frac{0}{2}}{\frac{0}{0}}$} & 0 & 16.20 & 46.60 & 22.48 & 46.60 & 2.43 \\
\hline & & 1 & 18.00 & 51.51 & 25.27 & 51.51 & 2.70 \\
\hline & & 3 & 28.00 & 139.26 & 43.83 & 139.26 & 4.20 \\
\hline & & 5 & 22.00 & 121.10 & 35.03 & 121.10 & 3.30 \\
\hline & & 7 & 15.60 & 99.87 & 26.23 & 99.87 & 2.34 \\
\hline & \multirow{4}{*}{ 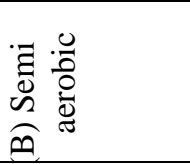 } & 1 & 28.67 & 117.07 & 43.98 & 117.07 & 4.30 \\
\hline & & 3 & 35.27 & 140.80 & 55.42 & 140.80 & 5.29 \\
\hline & & 5 & 33.67 & 152.38 & 53.14 & 152.38 & 5.05 \\
\hline & & 7 & 22.80 & 134.49 & 37.76 & 134.49 & 3.42 \\
\hline \multirow{9}{*}{ 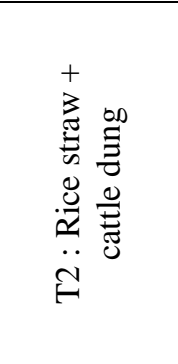 } & \multirow{5}{*}{$\underset{\frac{\pi}{2}}{\frac{0}{0}}$} & 0 & 16.67 & 108.60 & 27.27 & 108.60 & 2.50 \\
\hline & & 1 & 20.73 & 130.18 & 34.06 & 130.18 & 3.11 \\
\hline & & 3 & 32.87 & 235.77 & 55.29 & 235.77 & 4.93 \\
\hline & & 5 & 25.13 & 179.10 & 42.63 & 179.10 & 3.77 \\
\hline & & 7 & 16.80 & 138.16 & 29.21 & 138.16 & 2.52 \\
\hline & \multirow{4}{*}{ 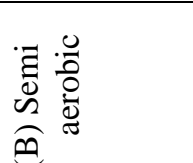 } & 1 & 35.47 & 118.99 & 54.48 & 118.99 & 5.32 \\
\hline & & 3 & 37.87 & 162.01 & 62.80 & 162.01 & 5.68 \\
\hline & & 5 & 35.80 & 177.46 & 61.01 & 177.46 & 5.37 \\
\hline & & 7 & 34.40 & 201.96 & 58.76 & 201.96 & 5.16 \\
\hline \multirow{9}{*}{ 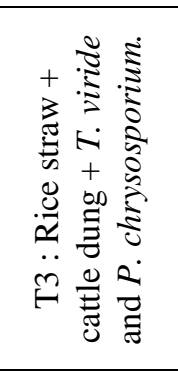 } & \multirow{5}{*}{ ङ $\frac{0}{0}$} & 0 & 19.40 & 94.91 & 30.45 & 94.91 & 2.91 \\
\hline & & 1 & 21.13 & 100.83 & 33.50 & 100.83 & 3.17 \\
\hline & & 3 & 34.13 & 179.27 & 55.18 & 179.27 & 5.12 \\
\hline & & 5 & 25.60 & 171.97 & 43.01 & 171.97 & 3.84 \\
\hline & & 7 & 17.07 & 110.54 & 29.52 & 110.54 & 2.56 \\
\hline & \multirow{4}{*}{ 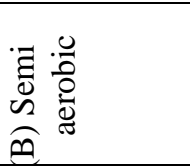 } & 1 & 38.07 & 147.62 & 60.02 & 147.62 & 5.71 \\
\hline & & 3 & 42.60 & 202.15 & 71.26 & 202.15 & 6.39 \\
\hline & & 5 & 38.33 & 212.65 & 66.57 & 212.65 & 5.75 \\
\hline & & 7 & 36.20 & 241.55 & 62.89 & 241.55 & 5.43 \\
\hline \multirow{9}{*}{ 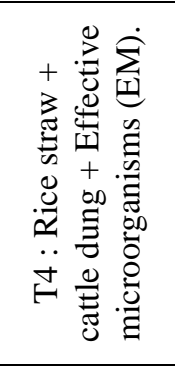 } & \multirow{5}{*}{$\underset{\frac{\pi}{2}}{\frac{0}{0}}$} & 0 & 20.13 & 67.17 & 29.54 & 67.17 & 3.02 \\
\hline & & 1 & 29.80 & 139.82 & 47.21 & 139.82 & 4.47 \\
\hline & & 3 & 39.60 & 220.24 & 64.35 & 220.24 & 5.94 \\
\hline & & 5 & 30.67 & 185.19 & 50.57 & 185.19 & 4.60 \\
\hline & & 7 & 17.20 & 269.59 & 31.14 & 269.59 & 2.58 \\
\hline & \multirow{4}{*}{ 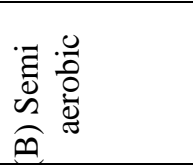 } & 1 & 52.80 & 335.03 & 92.40 & 335.03 & 7.92 \\
\hline & & 3 & 62.47 & 381.83 & 112.63 & 381.83 & 9.37 \\
\hline & & 5 & 53.40 & 331.81 & 96.53 & 331.81 & 8.01 \\
\hline & & 7 & 46.07 & 309.45 & 83.85 & 309.45 & 6.91 \\
\hline \multirow{3}{*}{$\begin{array}{l}\text { L.S.D } \\
(0.05)\end{array}$} & \multicolumn{2}{|c|}{ Treatment } & 0.2300 & 0.2542 & 0.2525 & 0.2542 & 0.0596 \\
\hline & \multirow{2}{*}{\multicolumn{2}{|c|}{$\begin{array}{l}\text { Pretreatment } \\
\text { Period of day }\end{array}$}} & 0.1627 & 0.1797 & 0.1785 & 0.1797 & 0.0421 \\
\hline & & & 0.7274 & 0.8038 & 0.7985 & 0.8038 & 0.1884 \\
\hline
\end{tabular}


Table 6. Methane production during different pretreatment processes of rice straw and anaerobic digestion at 50

\begin{tabular}{|c|c|c|c|c|c|c|c|c|}
\hline \multirow[b]{2}{*}{ Treatments } & \multirow[b]{2}{*}{ Conditions } & \multirow[b]{2}{*}{ 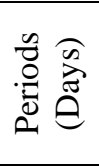 } & \multicolumn{6}{|c|}{ Methane production } \\
\hline & & & $\begin{array}{c}\% \text { of } \\
\text { biogas } \\
\text { produced }\end{array}$ & $\begin{array}{l}\text { Total } \\
\text { Liter }\end{array}$ & $\begin{array}{c}\text { TS } \\
\text { added } \\
(\mathrm{L} / \mathrm{Kg}) \\
\end{array}$ & $\begin{array}{c}\text { TS } \\
\text { consumed } \\
(\mathrm{L} / \mathrm{Kg}) \\
\end{array}$ & $\begin{array}{c}\mathrm{VS} \\
\text { added } \\
(\mathrm{L} / \mathrm{Kg})\end{array}$ & $\begin{array}{c}\mathrm{VS} \\
\text { consumed } \\
(\mathrm{L} / \mathrm{Kg}) \\
\end{array}$ \\
\hline \multirow{9}{*}{ 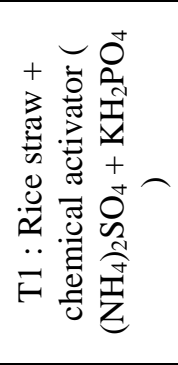 } & \multirow{5}{*}{ ¿ $\frac{0}{0}$} & 0 & 36.42 & 0.99 & 6.60 & 18.98 & 9.16 & 18.98 \\
\hline & & 1 & 43.21 & 1.28 & 8.53 & 24.42 & 11.98 & 24.42 \\
\hline & & 3 & 47.47 & 2.14 & 14.27 & 70.95 & 22.33 & 70.95 \\
\hline & & 5 & 44.99 & 1.62 & 10.80 & 59.45 & 17.20 & 59.45 \\
\hline & & 7 & 36.19 & 0.95 & 6.33 & 40.55 & 10.65 & 40.55 \\
\hline & \multirow{4}{*}{ 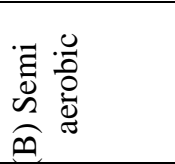 } & 1 & 45.42 & 2.05 & 13.67 & 55.81 & 20.97 & 55.81 \\
\hline & & 3 & 53.52 & 2.95 & 19.67 & 78.52 & 30.90 & 78.52 \\
\hline & & 5 & 50.45 & 2.70 & 18.00 & 81.47 & 28.41 & 81.47 \\
\hline & & 7 & 41.91 & 1.52 & 10.13 & 59.77 & 16.78 & 59.77 \\
\hline \multirow{9}{*}{ 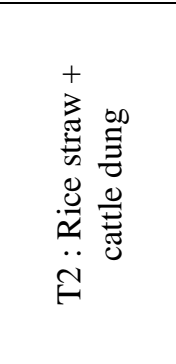 } & \multirow{5}{*}{ ङ $\frac{0}{0}$} & 0 & 37.06 & 1.07 & 7.13 & 46.48 & 11.67 & 46.48 \\
\hline & & 1 & 43.70 & 1.53 & 10.20 & 64.04 & 16.75 & 64.04 \\
\hline & & 3 & 47.88 & 2.58 & 17.20 & 123.39 & 28.94 & 123.39 \\
\hline & & 5 & 45.74 & 1.88 & 12.53 & 89.31 & 21.26 & 89.31 \\
\hline & & 7 & 36.89 & 1.03 & 6.87 & 56.47 & 11.94 & 56.47 \\
\hline & \multirow{4}{*}{ 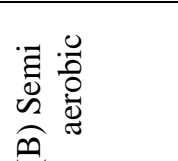 } & 1 & 46.30 & 2.60 & 17.33 & 58.15 & 26.63 & 58.15 \\
\hline & & 3 & 52.24 & 3.23 & 21.53 & 92.13 & 35.71 & 92.13 \\
\hline & & 5 & 51.26 & 2.92 & 19.47 & 96.50 & 33.17 & 96.50 \\
\hline & & 7 & 42.68 & 2.31 & 15.40 & 90.41 & 26.31 & 90.41 \\
\hline \multirow{9}{*}{ 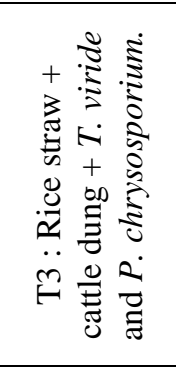 } & \multirow{5}{*}{ 度 $\frac{0}{0}$} & 0 & 42.21 & 1.31 & 8.73 & 42.73 & 13.71 & 42.73 \\
\hline & & 1 & 44.03 & 1.54 & 10.27 & 48.98 & 16.28 & 48.98 \\
\hline & & 3 & 48.61 & 2.71 & 18.07 & 94.89 & 29.21 & 94.89 \\
\hline & & 5 & 46.50 & 1.97 & 13.13 & 88.22 & 22.07 & 88.22 \\
\hline & & 7 & 37.49 & 1.06 & 7.07 & 45.77 & 12.22 & 45.77 \\
\hline & \multirow{4}{*}{ 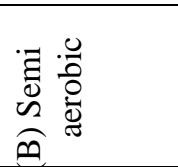 } & 1 & 46.99 & 2.79 & 18.60 & 72.13 & 29.33 & 72.13 \\
\hline & & 3 & 54.87 & 3.61 & 24.07 & 114.20 & 40.26 & 114.20 \\
\hline & & 5 & 52.10 & 3.16 & 21.07 & 116.86 & 36.59 & 116.86 \\
\hline & & 7 & 43.71 & 2.51 & 16.73 & 111.65 & 29.07 & 111.65 \\
\hline \multirow{9}{*}{ 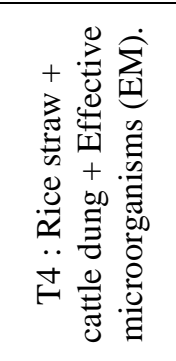 } & \multirow{5}{*}{ ङ $\frac{0}{0}$} & 0 & 43.17 & 1.38 & 9.20 & 30.69 & 13.50 & 30.69 \\
\hline & & 1 & 44.63 & 2.18 & 14.53 & 68.19 & 23.02 & 68.19 \\
\hline & & 3 & 49.10 & 3.22 & 21.47 & 119.39 & 34.88 & 119.39 \\
\hline & & 5 & 47.10 & 2.39 & 15.93 & 96.22 & 26.28 & 96.22 \\
\hline & & 7 & 38.28 & 1.09 & 7.27 & 113.90 & 13.15 & 113.90 \\
\hline & \multirow{4}{*}{ 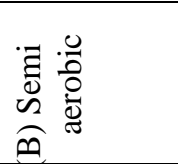 } & 1 & 47.51 & 3.98 & 26.53 & 168.36 & 46.44 & 168.36 \\
\hline & & 3 & 55.43 & 5.42 & 36.13 & 220.86 & 65.15 & 220.86 \\
\hline & & 5 & 52.81 & 4.42 & 29.47 & 183.10 & 53.27 & 183.10 \\
\hline & & 7 & 44.49 & 3.18 & 21.20 & 142.41 & 38.59 & 142.41 \\
\hline \multirow{3}{*}{$\begin{array}{l}\text { L.S.D } \\
(0.05)\end{array}$} & \multicolumn{2}{|c|}{ Treatment } & 0.2500 & 0.0374 & 0.2129 & 0.2214 & 0.4129 & 0.2214 \\
\hline & \multirow{2}{*}{\multicolumn{2}{|c|}{$\begin{array}{l}\text { Pretreatment } \\
\text { Period of day }\end{array}$}} & 0.1767 & 0.0265 & 0.1505 & 0.1566 & 0.2917 & 0.1566 \\
\hline & & & 0.7904 & 0.1184 & 0.6732 & 0.7002 & 1.3045 & 0.7002 \\
\hline
\end{tabular}

\section{Evaluation of biogas manure production}

Data presented in Table (7) show the evolution of biogas manure production by extraction of humic substances and their content of humic and fulvic acids as percentage of the equal total organic matter. Data indicated that humic acids concentrations were higher than fulvic acids in all treatments. The percentages of humic substances and humic acid obtained the best values with the treatment T4B3 where recorded 41.87 and $29.67 \%$, respectively. On the contrary, the treatment T1A7 achieved the worst values where recorded 19.89 and $11.21 \%$, respectively. The percentages of fulvic acid content of different biogas manure types and compost ranged from 8.66 to
$15.30 \%$. The acquired results are in agreement with those reported by Barje et al.(2012), found that through composting, low molecular weight compounds are polymerized to form HS which primarily contains two fractions of fulvic and humic acids. Junqiu et al. (2019) reported that humic substances and humic acids concentration were increased, even though fulvic acids concentration were decreased during composting. Zhou et al. (2014) has pointed that fulvic acids is mostly transformed to humic acids, resulting in the aromatization of humic substances, promoting the compost maturity, leading to the increase of humic substances and humic acids concentration and decrease of fulvic acids 
concentration. Clapp et al. (1993) reported that humic substances are natural organic compounds comprising from 50 to $90 \%$ of the organic matter of peat, lignites saproples and composts.

Table 7. Percentage of humic substances, humic and fulvic acid isolated from biogas manure

\begin{tabular}{|c|c|c|c|c|c|}
\hline Treatments & Conditions & $\begin{array}{c}\text { Periods } \\
\text { (Days) }\end{array}$ & Humic substances (\%) & Humic acid (\%) & $\begin{array}{l}\text { Fulvic acid } \\
\qquad(\%)\end{array}$ \\
\hline \multirow{9}{*}{ 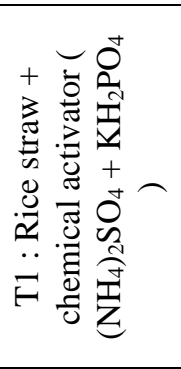 } & \multirow{5}{*}{$\underset{\&}{\frac{0}{0}} \frac{0}{0}$} & 0 & 24.86 & 13.17 & 11.69 \\
\hline & & 1 & 26.11 & 14.66 & 11.45 \\
\hline & & 3 & 28.35 & 15.16 & 13.19 \\
\hline & & 5 & 28.01 & 15.01 & 13.00 \\
\hline & & 7 & 19.89 & 11.21 & 8.68 \\
\hline & \multirow{4}{*}{ 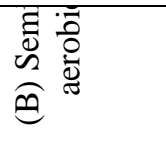 } & 1 & 28.91 & 17.05 & 11.86 \\
\hline & & 3 & 33.71 & 21.17 & 12.54 \\
\hline & & 5 & 30.86 & 19.73 & 11.33 \\
\hline & & 7 & 22.91 & 13.92 & 8.99 \\
\hline \multirow{9}{*}{ 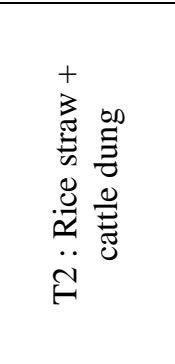 } & \multirow{5}{*}{$\underset{\frac{0}{0}}{\stackrel{0}{0}} \frac{0}{\frac{0}{4}}$} & 0 & 25.18 & 16.41 & 8.77 \\
\hline & & 1 & 27.18 & 17.91 & 9.27 \\
\hline & & 3 & 30.04 & 20.01 & 10.03 \\
\hline & & 5 & 29.66 & 18.13 & 11.53 \\
\hline & & 7 & 22.69 & 14.03 & 8.66 \\
\hline & \multirow{4}{*}{ 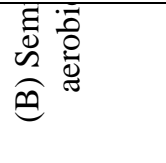 } & 1 & 33.21 & 20.03 & 13.18 \\
\hline & & 3 & 37.11 & 24.18 & 12.93 \\
\hline & & 5 & 34.71 & 23.03 & 11.68 \\
\hline & & 7 & 28.19 & 17.97 & 10.22 \\
\hline \multirow{9}{*}{ 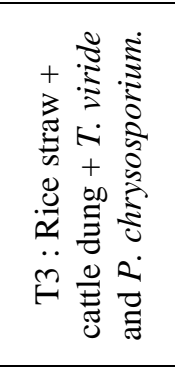 } & \multirow{5}{*}{$\underset{\ll}{\frac{0}{0}} \frac{0}{0}$} & 0 & 27.33 & 17.03 & 10.30 \\
\hline & & 1 & 29.34 & 19.41 & 9.93 \\
\hline & & 3 & 33.71 & 23.18 & 10.53 \\
\hline & & 5 & 31.17 & 20.16 & 11.01 \\
\hline & & 7 & 23.86 & 15.11 & 8.75 \\
\hline & \multirow{4}{*}{ 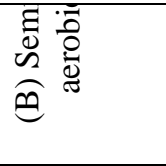 } & 1 & 35.18 & 21.31 & 13.87 \\
\hline & & 3 & 40.23 & 27.11 & 13.12 \\
\hline & & 5 & 38.17 & 25.11 & 13.06 \\
\hline & & 7 & 31.19 & 18.67 & 12.52 \\
\hline \multirow{9}{*}{ 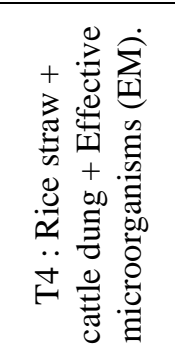 } & \multirow{5}{*}{$\underset{\frac{0}{4}}{\frac{0}{0}}$} & 0 & 29.21 & 18.96 & 10.25 \\
\hline & & 1 & 31.03 & 20.55 & 10.48 \\
\hline & & 3 & 35.55 & 26.11 & 9.44 \\
\hline & & 5 & 32.91 & 23.01 & 9.90 \\
\hline & & 7 & 26.31 & 16.41 & 9.90 \\
\hline & \multirow{4}{*}{ 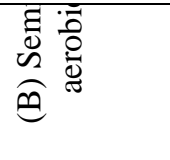 } & 1 & 38.69 & 23.41 & 15.28 \\
\hline & & 3 & 41.87 & 29.67 & 12.20 \\
\hline & & 5 & 40.05 & 27.31 & 12.74 \\
\hline & & 7 & 35.41 & 20.11 & 15.30 \\
\hline
\end{tabular}

\section{Conclusions}

From the abovementioned results, it has been concluded that the rice straw was promising for biogas production after semi aerobic pretreatment with effective microorganism, cattle dung where, this treatment gave the highest yield of biogas. Also, the best biogas manure contains much humic substances. In view of the obtained results, it could be recommended that use the application of effective microorganism, Tricoderma viride and Phanerochaete chrysosprium pretreatment (aerobic or semi aerobic) could be carried out before biogas production process.

\section{References}

Abdel-Fatah, W., E. (2012). Aerobic and anaerobic fermentation of rice straw with some microorganisms for production of biogas and compost. M.Sc., Thesis Fac. of Science, Tanta University.

ADP (2016). Bioconversion of agricultural residues in to compost for improving crop productively and environmental protection project 2015-2016.

Ali, L. K. M. (2011). A study of some methods of waste management of rice through its impact on soil physical properties, $\mathrm{N}, \mathrm{P}$ and $\mathrm{K}$ contents in maize yield and water use efficiency under different tillage systems, Australian J. of Basic and Applied Sci., 5 (5): 1017-1034. 
Analytical software (2013). STATISTIX 10 for windows. Analytical software, Tallahassee, FL, USA.

APHA; American Publich Health, Association. (1992). Standard methods for the examination of water and wastewater. $18^{\text {th }}$, Washington, D.C.

Ariunbaatar, J.; Panico, A.; Esposito, G.; Pirozzi, F. and Lens, P. N. L. (2014). Pretreatment methods to enhance anaerobic digestion of organic solid waste. Applied Energy, 123: 143-156.

Awe, O.W.; Zhao, Y.; Nzihou, A.; Minh, D. P. and Lyczko, N. (2017). A Review of Biogas Utilization, Purification and Upgrading Technologies. Waste Biomass Valor, 8: 267-283.

Barje, F.; El Fels, L.; El Hajjouji, H.; Amir, S.; Winterton, P. and Hafidi, M. (2012). Molecular behaviour of humic acid-like substances during co-composting of olive mill waste and the organic part of municipal solid waste. Int. Biodeterior. Biodegrad., 74: 17-23.

Budiyono, I. N.; Johari, S. and Sunarso, (2010). The influence of total solid contents on biogas yield from cattle manure using rumen fluid inoculum. Energy Res. J., 1 (1): 6-11.

Clapp, C. E.; Hayes, M. H. B. and Swift, R. S. (1993). Isolation, fractionation, functionalities, and concepts of structure of soil organic macromolecules, In: “Organic Substances in Soil and Water, Royal Society of Chemistry, Cambridge." (Beck, A. J.; Jones, K.C; Hayes, M. B. H. and Mingelgrin, U. eds.), BioAg Technologies International, 916: 371- 694.

Cochran, W. G. (1950). Estimation of bacteria densities by means of the " most probable number. "Biometrics, 6:105-116.

Cunningham, A. (1954). Practical Bacteriology. $2^{\text {nd }}$ Ed. Oliver and Boyed, Edinburgh and London.

Difco, M. (1977). Microbiological Laboratory procedures. Ninth Edition. Difco Laboratories, Detroit. Michigan, U.S.A. 855 p.

El-Akshar, Y. S. (2000). Microbiological studies on anaerobic digestion of solid and liquid wastes. M.Sc., Thesis Fac. of Agric., Moshtohor, Zagazig University.

El-Akshar, Y.S.; Estefanous, A.N and Afifi, M.M.I. (2012). The Influence of microbial inoculants on microbial activity and humic substances of rice straw composting. Res. J. Agric. and Biolo. Sci., 8, 2: 68-77.

Estefanous, A.N. (1993). Anaerobic degradation of high solid organic wastes to methane and organic fertilizers. Ph.D . Thesis, Fac. Agric., Moshtohor, Banha Branch, Zagazig Uni., Egypt.

Estefanous, A.N.; El-Akshar,Y.S.; El-Sayed, G.A.M. and Amal, A.Hana. (2010). Anaerobic digestion of municipal solid waste to produce biogas and organic manure by using leachate recycling. Egypt. J. Biotechnol., 36: $65-80$.

Hanafy, E., A.; Estefanous, R.A.; Zaghloul,R.A. and El-Aksher, Y.S. (2000). Biogas production from Jew's Mallow processing wastes and cattle dung using batch feeding system .Proceeding of Tenth Microbiol. Conference, Cairo, Egypt, 1114 Nov200.

Jodice, R.; Luzzati, A. and Nappi, P. (1982). The influence of organic fertilizers, obtained from poplar barks, on the correction of iron chlorosis of Luipinus albusl. Plant and Soil, 65: 309 - 317.

Jönsson, L.J. and Martin, C. (2016). Pretreatment of lignocellulose: Formation of inhibitory byproducts and strategies for minimizing their effects. Biores. Technol., 199: 103-112.

Junqiu, W.; Yue, Z.; Huimin, Y.; Dan, W.; Tianxue, Y.; Zimin, W.; Qian, L. and Xu, Z. (2019). Effects of aeration rates on the structural changes in humic substance during co-composting of digestates and chicken manure. Sci. of The Total Envir., 658: 510-520.

Lim, J. W.; Ge, T. and Tong, Y. W. (2018). Monitoring of microbial communities in anaerobic digestion sludge for biogas optimization. Waste Manag., 71: 334-341.

Liu, D.; Yan, X.; Zhuo, S. N.; Si, M. Y.; Liu, M. R.; Wang, S.; Ren, L. L.; Chai, L. Y. and Shi, Y. (2018). Pandoraea sp. B-6 assists the deep eutectic solvent pretreatment of rice straw via promoting lignin depolymerization. Biores. Technol., 257: 62-68.

MALR. (2015). Ministry of Agriculture and Reclamation. Economic Affaris Sector. Bulletin of the Agricultural statistics.

Maramba, F. D. ; Obias, E. D. ; Julian, B.; Taganas, C.; Alumbro, R. D. and Judan, A. A. (1978). Biogas and waste recycling, the Philippine experience. Maya farms division, liberity flour mills, Inc. Metro Manila, Philippines.

Maria, L.V.; Daniel, S.; Affonso, C. G. Jr.; Alfredo, R.; Jéssica, M.; Andréiada, P. S. and Thiago, B. S. (2019). Production of biogas and biofertilizer using anaerobic reactors with swine manure and glycerin doses. J. of Cleaner Production, 213: 176184.

Page, A. L.; Miller, R. H. and Keeney, D.R. (1982). Methods of Soil Analysis. Part 2. Soil Soc. Amer. Inc. Madison, Wisconsin, U.S.A., 310 p.

Rama, T. R. (2013). Separation of some rice straw components and studying their effect on some hydrophysical properties of two different soils. J. of Enviro.Chem. Engine., (1) 728-735.

Richards, L. A. (1954). Diagnosis and Improvement of Saline and Alkali Soil. U.S. Dept. Agric., 60: 50-75.

Sheldon , M. S. ; Mohammed, K. and Ntwampe, S. K. O. (2008). An investigation of biphasic growth kinetics for Phanerochaete chrysosporium (BKMF-1767) immobilised in a membrane gradostat reactor using flow-cells. Enzyme and Microbial Technol. 42: 353-361. 
Snedecor, G. W. and Cochran, W. G. (1991). Statistical Method 7 th Ed Iowa State Univ. Press, Iowa, USA.

Tom Volk. (2004). Trichoderma viride, the dark green parasitic mold and maker of fungal-digested jeans. Tom Volk's Fungus of the Month.

Ugwuoke, N. P.; Ude. M. U.; Ofili, I. and Abur, F. F. (2015). The effect of total solid concentration on biogas production. International J. of Sci., Engine. and Technolo. Res., 4, (9): 3096-3099.

Wujick, W. J. and Jewell, W. J. (1980). Dry anaerobic fermentation. Biotechnology and Bioengineering Symp., 10: 43-65. Jon Willey \&Sons, Inc. N.Y.

Yıldırım, E.; Ince, O.; Aydin, S. and Ince, B. (2017). Improvement of biogas potential of anaerobic digesters using rumen fungi. Renewable Energy, 109: 346-353.

Zhang, L.; Sun, X.; Tian, Y. and Gong, X. (2014). Biochar and humic acid amendments improve the quality of composted green waste as a growth medium for the ornamental plant Calathea insignis. Sci. Hortic., 176: 70-78.

Zhou, S. X.; Dong, Y. P. and Zhang, Y. L. (2012). Solid-state anaerobic digestion for methane production from corn stalks with stack-pretreated. Materials Sci. Forum, 697: 326-330.

Zhou, Y.; Selvam, A. and Wong, J.W.C. (2014). Evaluation of humic substances during cocomposting of food waste, sawdust and Chinese medicinal herbal residues. Biores. Technol., 168: 229-234.

\section{إنتاج البيوجاز بإستخدام المعاملات الأولية المختلفة لمخلفات قش الأرز تحت الظروف الهوائية و النصف هوائية.

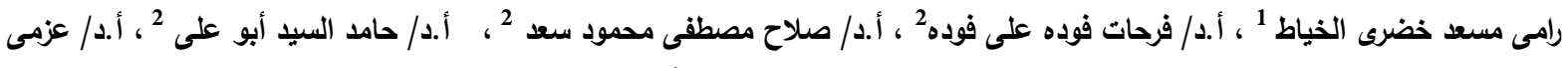

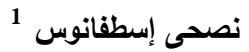

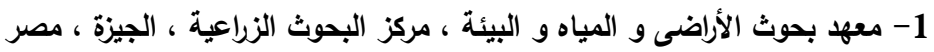

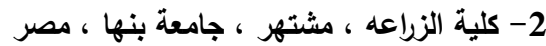

\footnotetext{
يعتبر قش الأرز من المخلفات النى تسبب مشاكل بيئية و ذلك لإنتثارها على مستوى الجمهورية و تأثيره كملوث بيئى للهواء نتيجة لإحتراقه و قلذة إستغلاله و تسببه فى البقعه السوداء لذا تهذف هذه الدراسة إلى إمكانية إستغلال مخلفات قش الأرز كمصدر للمادة العضوية لإنتاج البيوجاز و

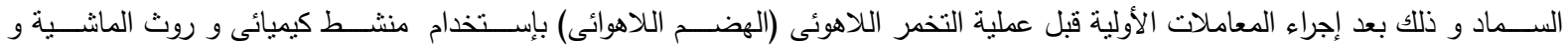

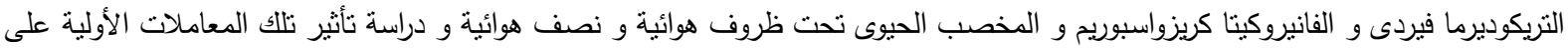

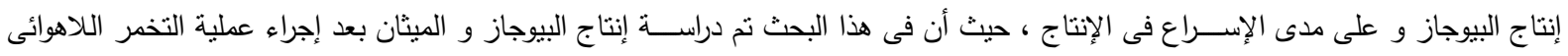

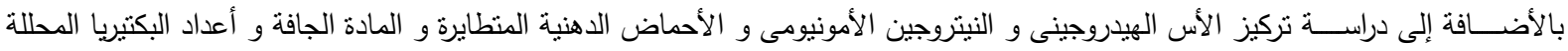

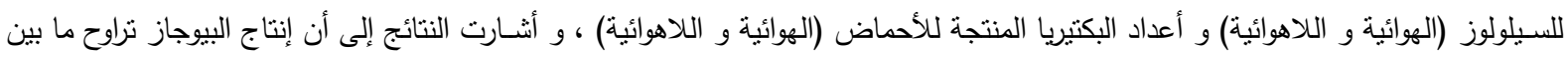

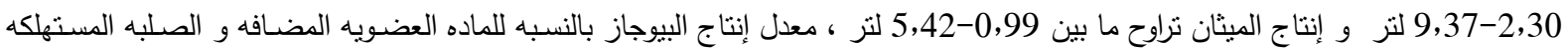

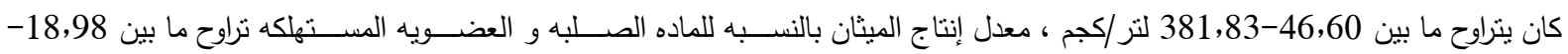

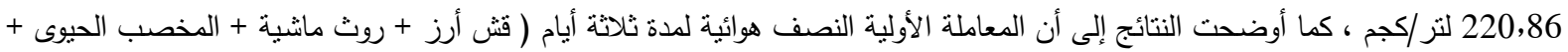

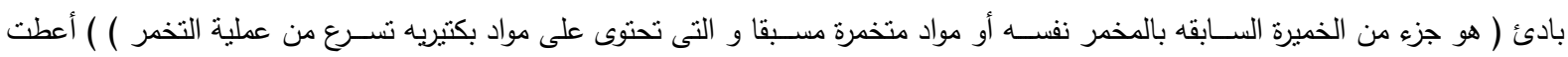

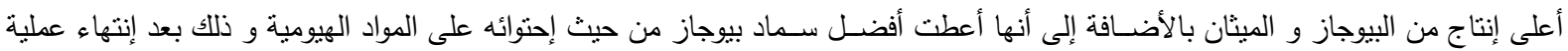

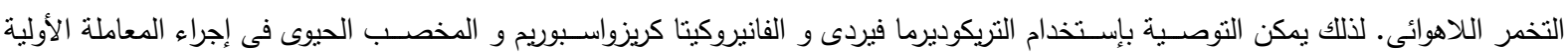
النصف هوائية لقش الأرز قبل عملية إنتاج البيوجاز (التخمر اللاهوائى).
} 